\title{
Pediatric COVID-19 and the Factors That May Mitigate Its Clinical Course
}

\author{
Mosaad Abdel-Aziz ${ }^{1}$ Nada M. Abdel-Aziz ${ }^{2}$ Dina M. Abdel-Aziz ${ }^{3}$ Noha Azab ${ }^{4}$ \\ ${ }^{1}$ Department of Otolaryngology, Faculty of Medicine, Cairo \\ University, Cairo, Egypt \\ 2 Faculty of Dentistry, October University of Modern Science and Arts \\ (MSA), October City, Egypt

\begin{abstract}
Address for correspondence Mosaad Abdel-Aziz, MD, Department of Otolaryngology, Faculty of Medicine, Cairo University, 2 el-salam Street, King Faisal, Above el-baraka Bank, Giza, Cairo 12111, Egypt (e-mail: mosabeez@yahoo.com).
\end{abstract}

3 Faculty of Medicine, Misr University for Science and Technology (MUST), Misr, Egypt

4 Department of Rheumatology and Rehabilitation, Faculty of Medicine, Cairo University, Cairo, Egypt

J Child Sci 2020;10:e137-e140.

\begin{abstract}
Keywords

- coronavirus

- COVID-19

- SARS-CoV-2 infection

- pediatric infection

- trained immunity

The clinical manifestations of novel coronavirus disease 2019 (COVID-19) vary from mild flu-like symptoms to severe fatal pneumonia. However, children with COVID-19 may be asymptomatic or may have mild clinical symptoms. The aim of this study was to investigate clinical features of pediatric COVID-19 and to search for the factors that may mitigate the disease course. We reviewed the literature to realize the clinical features, laboratory, and radiographic data that may be diagnostic for COVID-19 among children. Also, we studied the factors that may affect the clinical course of the disease. Fever, dry cough, and fatigue are the main symptoms of pediatric COVID-19, sometimes flu-like symptoms and/or gastrointestinal symptoms may be present. Although some infected children may be asymptomatic, a recent unusual hyperinflammatory reaction with overlapping features of Kawasaki's disease and toxic shock syndrome in pediatric COVID-19 has been occasionally reported. Severe acute respiratory syndrome-coronvirus-2 (SARS-CoV-2) nucleic acid testing is the cornerstone method for the diagnosis of COVID-19. Lymphocyte count and other inflammatory markers are not essentially diagnostic; however, chest computed tomography is highly specific. Factors that may mitigate the severity of pediatric COVID-19 are home confinement with limited children activity, trained immunity caused by compulsory vaccination, the response of the angiotensin-converting enzyme 2 receptors in children is not the same as in adults, and that children are less likely to have comorbidities. As infected children may be asymptomatic or may have only mild respiratory and/or gastrointestinal symptoms that might be missed, all children for families who have a member diagnosed with COVID-19 should be investigated.
\end{abstract}

\section{Introduction}

Coronavirus is a highly contagious pathogen which mainly affects the respiratory system. Previous outbreaks of this virus include the severe acute respiratory syndrome (SARS-
$\mathrm{CoV}$ ) and the Middle East respiratory syndrome (MERS-CoV) which caused major impact on the public health. In December 2019, many patients were hospitalized in Wuhan, China, with pneumonia of an unknown cause. ${ }^{1}$ The offending pathogen was identified and nominated as 2019 novel received

July 24,2020

accepted after revision

August 20, 2020
DOI https://doi.org/

10.1055/s-0040-1717077. ISSN 2474-5871.

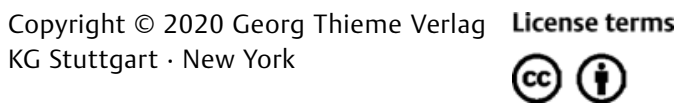


coronavirus (2019-nCoV) which is a new coronavirus that has never been detected in humans before. ${ }^{2}$ Since then, the number of patients has increased dramatically, and the World Health Organization (WHO) named the virus as SARS-CoV-2 and the illness as novel coronavirus disease 2019 (COVID-19) on February 11, 2020. WHO has declared the outbreak as a pandemic disease on March 11, 2020. ${ }^{3}$

The clinical manifestations of COVID-19 vary from mild flu-like symptoms to severe fatal pneumonia, fortunately the commonest presentation is the mildest form of the disease. ${ }^{4}$ Indeed, most published data showed that the disease has a mild clinical course and a better prognosis in children. ${ }^{5}$ However, an unusual hyperinflammatory illness with overlapping features of Kawasaki's disease (KD) and toxic shock syndrome in children with COVID-19 has been detected. ${ }^{6}$ Although this pediatric inflammatory multisystem syndrome is rarely reported to be associated with SARS-CoV-2 infection, it caused a major concern. ${ }^{7}$ Literature from China, Italy, and the United States reported a pediatric incidence of COVID-19 that ranged from 1.2 to $5 \%$ of infected population. ${ }^{3}$ The disease is usually transmitted by droplet infection, and the infection source of children is often one of the adult family members, with an incubation period ranging from 2 to 10 days. $^{5,8}$ As pediatric patients are usually vulnerable to respiratory infections, and as COVID-19 among them may have a milder course that may be similar to common cold, ${ }^{9}$ delayed diagnosis of COVID-19 among children may be a problem. The objective of this study was to investigate the clinical manifestations, diagnostic criteria, and the factors that may mitigate its clinical course among pediatric patients.

\section{Methods}

A thorough review of PubMed, Science Direct, and Web of Science of articles published until July 2020 was conducted; using the keywords COVID-19, pediatrics, children, severity, and laboratory and radiographic findings. No language restrictions were applied. All articles were evaluated for inclusion and screened by titles and abstracts for relevance and possible eligibility. Due to the increasing numbers of papers on pediatric COVID-19, studies were deemed eligible for inclusion if they were closely related to our topic either review articles, original studies, case series, or case reports. Articles that report clear and extractable data on clinical, laboratory, and radiographic findings of pediatric COVID-19 were included. Articles with reiterated data were excluded, so we did not assess the risk of bias or grade the level of the generated evidence.

\section{Clinical Manifestations}

The most common clinical manifestations of COVID-19 are fever, fatigue, cough, and expectoration; other symptoms included myalgia, anorexia, chest tightness, shortness of breath, and dyspnea. ${ }^{10,11}$ However, infected children may look asymptomatic or may have mild noncharacteristic symptoms, such as dry cough, mild fever, and fatigue, as well as upper respiratory infection symptoms, including nasal obstruction and running nose. Also, some patients may present with gastrointestinal (GIT) symptoms as abdominal pain, nausea, vomiting, and diarrhea. ${ }^{3,9}$ However, children may present with upper respiratory infection and/or diarrhea only, and the travel history of a family member may raise the suspicion of diagnosis for COVID$19 .^{12}$ Indeed, clinically asymptomatic children who had a positive SARS-CoV-2 test have been reported. ${ }^{13}$ On the other hand, severe pneumonia with dyspnea, cyanosis, and systemic toxic manifestations as malaise, restlessness, loss of appetite, poor feeding, and less activity have also been reported as a presentation in some children. ${ }^{14,15}$ So, variability of clinical manifestations of the disease among pediatric patients should be expected. ${ }^{12}$ Adult and elderly patients usually present with more severe symptoms with an incidence of respiratory failure and/or acute respiratory distress syndrome of approximately $19.5 \% .{ }^{11}$ They may even develop septic shock, refractory metabolic acidosis, and coagulation dysfunction rapidly within 1 week. ${ }^{9}$ However, the development of these catastrophic complications with multiorgan dysfunction is rarely reported in children that usually have good prognosis and may recover within 1 to 2 weeks from disease onset. ${ }^{5-7,16}$

\section{Laboratory Evaluation}

SARS-CoV-2 nucleic acid testing is the corner-stone method for diagnosis of COVID-19. It can be detected by reverse transcriptase-polymerase chain reaction (RT-PCR) or through viral gene sequencing of throat swabs, sputum, stool, or blood samples. ${ }^{15}$ Adult patients usually show increased leukocyte and neutrophil counts with a significant decrease in the absolute number of lymphocytes at an early stage of the disease. In children, white blood cell count and absolute lymphocyte number are usually normal; however, lymphocyte depletion could occur at a later stage. ${ }^{17-19}$ Henry et al ${ }^{18}$ reported that the leucocyte count of children with COVID-19 was normal in $69.6 \%$, increased in $15.2 \%$, and decreased in $15.2 \%$; and the majority of them had normal neutrophil counts. Lymphopenia has been found by Wang et $\mathrm{al}^{20}$ in only $3 \%$ of pediatric patients, while it was found by Yang et $\mathrm{al}^{21}$ in $80 \%$ of adult patients; however, all children in the former study were asymptomatic or had mild manifestations and most adult patients in the latter study were critically ill. The lack of lymphopenia in children may be due to the mild form of the disease among this sector of patients, as it may correlate with severity of infection. ${ }^{18}$ However, lymphopenia may not occur in young children due to the relative immune system immaturity with differences in the immune response when compared with adults. ${ }^{14} \mathrm{C}$-reactive protein (CRP) and procalcitonin (PCT) which are inflammatory markers were noticed to be elevated in 13.6 and $10.6 \%$ of pediatric patients, respectively. However, in adult patients both CRP and PCT were seen elevated especially with unfavorable progression. ${ }^{18}$ Xia et al ${ }^{19}$ detected PCT elevation in $80 \%$ of pediatric patients, and they stressed on this important finding in the diagnosis; however, Chen et $\mathrm{al}^{15}$ reported that an elevated 
PCT level may indicate bacterial coinfection which is common in children. Also, severe cases may have elevated liver enzymes, muscle enzymes, myoglobin, and D-dimer. Serum ferritin has been reported to be highly elevated in children with severe form of the disease. ${ }^{22}$ Patients with hyperinflammatory response usually have elevated serum cytokines, especially tumor necrosis factor $\alpha$ (TNF $\alpha$ ) and interleukin- 6 (IL-6) that may indicate tissue damage., ${ }^{5,22}$

\section{Radiographic Examination}

As most of the children with COVID-19 have mild disease, plain chest X-ray may be invaluable, and chest computed tomography $(\mathrm{CT})$ is usually needed to identify the pulmonary lesions. ${ }^{2}$ However, 70 to $80 \%$ of pediatric patients may have radiographic lung abnormalities on chest CT. ${ }^{19,23}$ The lesions are usually subpleural with localized inflammatory infiltration which may be unilateral in approximately 30\% and bilateral in approximately $50 \%$ of patients. They may be in the form of consolidation with surrounding halo sign in $50 \%$, ground glass opacities in $60 \%$, fine mesh shadow in $20 \%$, and very small nodules in $15 \%{ }^{19}$ Li et $\mathrm{al}^{24}$ reported that CT can be helpful in the diagnosis of the disease as the current laboratory tests are time consuming and sometimes demonstrate false-negative results, and do not always keep up with the needs of a growing number of infected people.

\section{Discussion}

Fever, dry cough, and fatigue are the main symptoms of pediatric COVID-19, sometimes, flu-like and/or GIT symptoms may be present. However, some infected children may be asymptomatic. ${ }^{3}$ An important observation is that children who were diagnosed with COVID-19 had been infected from household family contacts. ${ }^{2}$ However, the population susceptible to infection is mainly older adults with low immunity, and there have been few reports of pediatric patients. ${ }^{24}$ Ludvigsson $^{3}$ reported that children represented $2 \%$ of diagnosed patients in China, $1.2 \%$ of patients in Italy, and $5 \%$ of COVID-19 positive cases in the United States. As the children may remain asymptomatic or even undiagnosed due to mild clinical symptoms, they might be considered a potential source of infection to their surrounding community. ${ }^{25}$

On the other hand, SARS-CoV-2 infection has been linked to a severe form of hyperinflammatory reactions in some pediatric patients. ${ }^{26}$ Recently, cases of a pediatric inflammatory multisystem syndrome with features mimicking KD have been reported in many countries. ${ }^{22,27} \mathrm{KD}$ is considered a main cause of acquired heart disease in developed countries. $^{22}$ Infectious agents could be the main trigger of KD, although no specific organism has been specified yet. ${ }^{28}$ However, host genetic factors may be involved in the pathophysiology of KD, which may result in overactivation of the innate immune system of the child. ${ }^{29,30}$

The rarity of the disease and its mild clinical course among most children may be attributed to different reasons. The decreased activities of children due to home confinement may have limited the disease spread. SARS-CoV-2 is one of the RNA viruses which are prone to errors in replication and mutation with lowering of their virulence. As children are mainly infected by their adult family members, they may be infected with the second, third, or even fourth generation of the virus, thereby get milder symptoms. ${ }^{2}$ Less dysfunction of the immune system of the children may be due to the theory of the trained immunity caused by the use of certain vaccines such as Bacille Calmette-Guerin (BCG) to train innate immunity to acquire immune memory. Most children of Asian countries received regular immunizations including BCG that has been proved to give nonspecific protection of mice against influenza virus infection, probably by the induction of trained immunity. So, the mild clinical symptoms of COVID-19 in children may be due to trained immunity. ${ }^{17}$ Also, some authors reported that patients who received measles, mumps, and rubella (MMR) vaccine showed reduced death rate from COVID$19 .{ }^{29}$ Adult patients with COVID-19 usually show a significant lymphopenia at an early stage of the disease, which is not the case in children; supporting the theory of trained immunity. ${ }^{2,17,25}$ It has been reported that entry of the SARSCoV-2 into the host cells is most likely via the angiotensinconverting enzyme 2 (ACE2) receptors which is similar to SARS-CoV. ${ }^{1}$ The number and/or the function of ACE2 receptors in children is not the same as in adults, which might explain the difference in severity of the disease. ${ }^{2}$ Furthermore, the respiratory mucosa of children may have many different types of viruses which may limit the growth of SARS-CoV-2. ${ }^{5}$ Also, we can't ignore that the children usually have less comorbidities than adults, with less smoking exposure, and they have more self-healing capacity. All these factors may decrease the severity of the clinical course of the COVID-19 in children.

Our study showed that the diagnosis of COVID-19 among the pediatric population is dependent mainly on detection of the viral nucleic acid either through viral gene sequencing of throat swabs, sputum, stool, or blood samples, or by RT-PCR. However, hematologic parameters may not be diagnostic and are usually related to the immune response against the viral infection. Chest CT may be helpful in the diagnosis of the disease as it may show abnormalities in 70 to $80 \%$ of cases. As the current laboratory tests may be not available, and they are time consuming and sometimes give false-negative results, the impact of chest $\mathrm{CT}$ increases as a diagnostic criterion for pediatric COVID-19. Especially, if the radiographic changes were associated with a history of contact of the child to a family member confirmed to have the disease. ${ }^{24}$

\section{Limitations}

A major limitation of our study is that the data of COVID-19 among pediatric population is insufficient, and that the literature is still inadequate to clarify all aspects of the disease. However, we have attempted to explain the characteristics of the disease in children and to present the factors that may lie behind the mild clinical form of the disease in children. 


\section{Conclusion}

Infection in children with SARS-CoV-2 is mostly transmitted from an infected family member. Infected children may be asymptomatic or may have only mild respiratory and/or GIT symptoms that might be missed. As the disease has a mild form among pediatric patients that may be undiagnosed, they may act as a potential source for spread of infection. Factors that may mitigate the severity of pediatric COVID-19 are as follows: home confinement with limited children activity, trained immunity caused by compulsory vaccination, the response of the ACE2 receptors is not the same as in adults, and that children are less likely to have comorbidities.

\section{Funding \\ None. \\ Conflict of Interest \\ None declared.}

\section{References}

1 Rothan HA, Byrareddy SN. The epidemiology and pathogenesis of coronavirus disease (COVID-19) outbreak. J Autoimmun 2020; 109:102433

2 Su L, Ma X, Yu H, et al. The different clinical characteristics of corona virus disease cases between children and their families in China - the character of children with COVID-19. Emerg Microbes Infect 2020;9(01):707-713

3 Ludvigsson JF. Systematic review of COVID-19 in children shows milder cases and a better prognosis than adults. Acta Paediatr 2020;109(06):1088-1095

4 Lake MA. What we know so far: COVID-19 current clinical knowledge and research. Clin Med (Lond) 2020;20(02):124-127

5 Haşlak F, Yıldız M, Adrovic A, Barut K, Kasapçopur Ö. Childhood rheumatic diseases and COVID-19 pandemic: an intriguing linkage and a new horizon. Balkan Med J 2020;37(04):184-188

6 Whittaker E, Bamford A, Kenny J, et al; PIMS-TS Study Group and EUCLIDS and PERFORM Consortia. Clinical characteristics of 58 children with a pediatric inflammatory multisystem syndrome temporally associated with SARS-COV-2. JAMA 2020

7 Ramcharan T, Nolan O, Lai CY, et al. Paediatric inflammatory multisystem syndrome: temporally associated with SARS-COV-2 (PIMS-TS): cardiac features, management and short-term outcomes at a UK tertiary paediatric hospital. Pediatr Cardiol 2020 (e-pub ahead of print) . Doi: 10.1007/s00246-020-02391-2

8 Cai J, Xu J, Lin D, et al. A case series of children with 2019 novel coronavirus infection: clinical and epidemiological features. Clin Infect Dis 2020 (e-pub ahead of print) . Doi: 10.1093/cid/ciaa198

9 Shen K, Yang Y, Wang T, et al; China National Clinical Research Center for Respiratory Diseases National Center for Children's Health, Beijing, China Group of Respirology, Chinese Pediatric Society, Chinese Medical Association Chinese Medical Doctor Association Committee on Respirology Pediatrics China Medicine Education Association Committee on Pediatrics Chinese Research Hospital Association Committee on Pediatrics Chinese Non-government Medical Institutions Association Committee on Pediatrics China Association of Traditional Chinese Medicine, Committee on Children's Health and Medicine Research China News of Drug Information Association, Committee on Children's Safety Medication Global Pediatric Pulmonology Alliance. Diagnosis, treatment, and prevention of 2019 novel coronavirus infection in children: experts' consensus statement. World J Pediatr 2020;16(03):223-231
10 Krajewska J, Krajewski W, Zub K, Zatoński T. COVID-19 in otolaryngologist practice: a review of current knowledge. Eur Arch Otorhinolaryngol 2020;277(07):1885-1897

11 Zhu J, Ji P, Pang J, et al. Clinical characteristics of 3062 COVID-19 patients: A meta-analysis. J Med Virol 2020 (e-pub ahead of print). Doi: $10.1002 / j m v .25884$

12 Ji LN, Chao S, Wang YJ, et al. Clinical features of pediatric patients with COVID-19: a report of two family cluster cases. World J Pediatr 2020;16(03):267-270

13 Chan JF, Yuan S, Kok KH, et al. A familial cluster of pneumonia associated with the 2019 novel coronavirus indicating person-toperson transmission: a study of a family cluster. Lancet 2020;395 (10223):514-523

14 Chen F, Liu ZS, Zhang FR, et al. [First case of severe childhood novel coronavirus pneumonia in China] (in Chinese). Zhonghua Er Ke Za Zhi 2020;58(03):179-182

15 Chen ZM, Fu JF, Shu Q, et al. Diagnosis and treatment recommendations for pediatric respiratory infection caused by the 2019 novel coronavirus. World J Pediatr 2020;16(03):240-246

16 Shen KL, Yang YH. Diagnosis and treatment of 2019 novel coronavirus infection in children: a pressing issue. World J Pediatr 2020;16(03):219-221

17 Cao Q, Chen YC, Chen CL, Chiu CH. SARS-CoV-2 infection in children: Transmission dynamics and clinical characteristics. J Formos Med Assoc 2020;119(03):670-673

18 Henry BM, Lippi G, Plebani M. Laboratory abnormalities in children with novel coronavirus disease 2019. Clin Chem Lab Med 2020;58(07):1135-1138

19 Xia W, Shao J, Guo Y, Peng X, Li Z, Hu D. Clinical and CT features in pediatric patients with COVID-19 infection: different points from adults. Pediatr Pulmonol 2020;55(05):1169-1174

20 Wang D, Ju XL, Xie F, et al. [Clinical analysis of 31 cases of 2019 novel coronavirus infection in children from six provinces (autonomous region) of northern China] (in Chinese). Zhonghua $\mathrm{Er}$ Ke Za Zhi 2020;58(04):269-274

21 Yang X, Yu Y, Xu J, et al. Clinical course and outcomes of critically ill patients with SARS-CoV-2 pneumonia in Wuhan, China: a single-centered, retrospective, observational study. Lancet Respir Med 2020;8(05):475-481

22 Pouletty M, Borocco C, Ouldali N, et al. Paediatric multisystem inflammatory syndrome temporally associated with SARS-CoV-2 mimicking Kawasaki disease (Kawa-COVID-19): a multicentre cohort. Ann Rheum Dis 2020;79(08):999-1006

23 Yang P, Liu P, Li D, Zhao D. Corona virus disease 2019, a growing threat to children? J Infect 2020;80(06):671-693

$24 \mathrm{Li} \mathrm{W}$, Cui H, Li K, Fang Y, Li S. Chest computed tomography in children with COVID-19 respiratory infection. Pediatr Radiol 2020;50(06):796-799

25 Cai JH, Wang XS, Ge YL, et al. [First case of 2019 novel coronavirus infection in children in Shanghai] (in Chinese). Zhonghua Er Ke Za Zhi 2020;58(02):86-87(in Chinese)

26 Haslak F, Yildiz M, Adrovic A, et al. Management of childhoodonset autoinflammatory diseases during the COVID-19 pandemic. Rheumatol Int 2020;40(09):1423-1431

27 Chiotos K, Bassiri H, Behrens EM, et al. Multisystem inflammatory syndrome in children during the covid-19 pandemic: a case series. J Pediatric Infect Dis Soc 2020;9(03):393-398

28 Kumrah R, Vignesh P, Rawat A, Singh S. Immunogenetics of Kawasaki disease. Clin Rev Allergy Immunol 2020;59(01): 122-139

29 Ebina-Shibuya R, Namkoong H, Shibuya Y, Horita N. Multisystem inflammatory syndrome in children (MIS-C) with COVID-19: insights from simultaneous familial Kawasaki disease cases. Int J Infect Dis 2020;97:371-373

30 Elakabawi K, Lin J, Jiao F, Guo N, Yuan Z. Kawasaki disease: global burden and genetic background. Cardiol Res 2020;11(01):9-14 